\title{
The adipose tissue/central nervous system axis
}

\author{
T.M. L oftus' ${ }^{1}$, D.G . Maggs' ${ }^{2}$ M. D. L ane ${ }^{1}$ \\ ${ }^{1}$ Department of Biological Chemistry, Johns Hopkins University School of Medicine, Baltimore, Maryland, USA \\ ${ }^{2}$ Endocrinology Division, Yale University School of Medicine, New Haven, Connecticut USA
}

Over the past five decades, evidence has accumulated to support the concept that adipose tissue mass is subject to homeostatic regulation. This concept is based on a model in which humoral signals generated in adipose tissue act in the brain to reduce food intake and promote weight loss [1], and that dysregulation of this system can result in obesity. The recent discovery of leptin [2] has generated enormous interest and important new advances in this rapidly evolving field. Leptin is a peptide secreted by adipose tissue that acts in the central nervous system in the region of the hypothalamus and perhaps elsewhere. Evidence would suggest that leptin functions as an essential afferent signal in the adipose tissue/central nervous system homeostatic loop (Fig. 1). This important discovery, and increasing information concerning the processing of afferent signals in the hypothalamus, raises the exciting prospect of the development of new therapeutic interventions that effectively reduce adiposity in obese humans. Since obesity and non-insulin-dependent diabetes are conditions that often co-exist, and since the prevalence of both conditions is increasing in westernized cultures, there is a compelling need for an improved understanding of the physiological and pharmacological aspects of the body weight regulatory system. This session focuses on the role of adipose tissue as a source of an important afferent signal (i.e. leptin) and the processing of this signal in the central nervous system.

Participants: J. Friedman, HHMI, The Rockefeller University, New York, New York, USA

M. Schwartz, Department of Veterans Affairs, Seattle, Washington, USA

Corresponding author: T. M. Loftus, Ph.D., Department of Biological Chemistry, Johns Hopkins University School of Medicine, 725 N. Wolfe Street, Baltimore, MD 21205, USA
Jeffrey Friedman: Leptin and its receptor

The initial identification of the gene encoding leptin resulted from studies of the ob/ob mutant mouse. Mice, homozygous for the obese (ob) mutation, demonstrate massive obesity, a reduced basal metabolic rate, hyperphagia, hyperglycaemia, hyperinsulinaemia, and reduced fertility [3]. Important insight into the role of this gene was provided by the classic parabiosis (cross-circulation) experiments of Coleman with ob/ob, db/db and lean mice [4]. These studies indicated that a factor, present in the blood of lean or $\mathrm{db} / \mathrm{db}$ obese mice, reversed the effects of the $o b$ mutation. Furthermore, they indicated that $d b / d b$ mice lacked the ability to respond to this factor. The positional cloning of the obese gene led to the identification of the obese gene product, leptin, a $16 \mathrm{kDa}$ protein that possesses a leader sequence and is secreted [2]. The obese gene of ob/ob mice possesses a mutation in the coding region that causes premature translational termination and gives rise to a truncated non-functional protein. Consistent with the hypothesis that ob/ob mice lack a blood-borne factor produced by lean and $\mathrm{db} / \mathrm{db}$ mice [4], administration of recombinant leptin to ob/ob mice causes a dramatic decrease in food intake and body weight.

Following identification of the leptin receptor, five receptor isoforms were described that arise through alternative splicing of the primary transcript. The five receptor isoforms possess identical $\mathrm{N}$-terminal ligand-binding domains, but differ in their C-terminal segments. The "long" form (obR-b) possesses a transmembrane-spanning segment and a large cytoplasmic domain of approximately 300 amino acids which contains JAK and STAT consensus binding sites. Three additional "short" isoforms (obR-a, c and d) have an alternate cytoplasmic domain lacking the STAT and one of the JAK sites found in the "long" form. The remaining form lacks a transmembrane domain and is 
presumably secreted. Preliminary studies support the presence of this form in mouse serum.

Leptin treatment of the $\mathrm{db} / \mathrm{db}$ obese mice failed to induce weight loss, suggesting that $\mathrm{db} / \mathrm{db}$ mice lack a functional leptin receptor. A mutation in the leptin receptor gene of the $\mathrm{db} / \mathrm{db}$ mouse generates a strong alternative splice donor which eliminates the splice variant that encodes the obR-b form of the receptor and instead gives rise to an alternatively spliced short form lacking most of the signal-transducing domain. Thus, blocking the "leptin signal" either by producing a non-functional leptin molecule (as in the ob/ob mouse) or by mutating the obR-b form of the receptor (as in the case of the $\mathrm{db} / \mathrm{db}$ mouse) has the same consequences suggesting that all or most of the effects of leptin on energy balance are mediated by this receptor isoform. In the hypothalamus, which is enriched for obR-b, leptin has been shown to activate STAT3 presumably via a JAK kinase. Tyrosine-phosphorylated STAT is translocated to the nucleus where it is thought to bind to a specific DNA sequence and activate genes important for energy homeostasis. No such activation was observed in other tissues in which the short forms of the receptor predominate. It should be noted that leptin receptor mutations have also been identified in $129^{3 \mathrm{j}}$ mice, fatty/corpulent rats and fa/fa fatty rats. Thus there are now four rodent models documented to develop obesity due to a primary leptin resistance syndrome.

It is likely that leptin plays different physiological roles depending on its level. Low levels of leptin may be perceived by the brain as starvation. Consistent with this, the anovulatory response observed in fasted mice is corrected by leptin administration in spite of continued caloric restriction. Low levels of leptin also lead to a strong hyperphagic response and a reduced metabolic rate. Thus the massive obesity, hyperphagia, and reproductive dysfunction in ob/ob mice may result from an attempt to compensate for a perceived fasted state. Conversely, high levels of leptin signal an energy surplus. Elevation of leptin levels by administration to rodents leads to a profound decrease in feeding and to adipose tissue loss without decreases in lean body mass or energy expenditure. This is in contrast to observations with calorically restricted animals which exhibit decreases in adipose mass, lean body mass, and energy expenditure.

As previously noted, leptin is expressed at exceptionally high levels in most obese humans (95\%). That obesity persists in spite of high levels of leptin, which should promote weight loss, implies that these individuals have acquired a resistance to the leptin signal. It is unknown whether this is a primary leptin resistance, i. e. a failure of leptin access to or signalling by its receptors, or a secondary resistance in which a superceding cause leads to obesity and gives rise to a compensatory elevation in leptin. Leptin resistance is also observed in several animal models of obesity with genetically normal leptin and leptin receptor genes. Agouti, fat, and tubby mice all exhibit elevated leptin levels coincident with obesity. AKR/J mice, when fed a normal diet, are lean and maintain normal leptin levels. However, upon feeding of a high fat diet, these animals become obese and express elevated levels of leptin. Correction of this obesity with leptin requires a ten times higher dose, consistent with an acquired form of leptin resistance. Interestingly, the New Zealand obese (NZO) mouse shows peripheral but not central resistance to leptin, suggesting that the blood brain barrier may play a role in leptin resistance in this model.

\section{Daniel Lane: Regulation of the leptin gene}

Tissue distribution studies revealed that the expression of leptin is adipose-specific. Transcription of the obese gene is activated during adipocyte differentiation. Kinetic studies, performed during differentiation of 3T3-L1 preadipocytes in culture, showed that expression of the leptin message is preceded by the expression of $\mathrm{C} / \mathrm{EBP} \alpha$ and that this occurs concomitantly with the expression of a representative adipocyte gene (the stearoyl-CoA desaturase-1 gene) whose promoter is known to be transcriptionally activated by $\mathrm{C} / \mathrm{EBP} \alpha$ [5]. $\mathrm{C} / \mathrm{EBP} \alpha$ is required for the expression of many adipocyte genes during differentiation. Consistent with this observation, the proximal promoter of the obese gene was found to contain several candidate C/EBP binding sites. DNase I footprinting of the proximal promoter by nuclear extracts from adipocytes (but not preadipocytes), demonstrated protection of a single region corresponding to a consensus $\mathrm{C} / \mathrm{EBP}$ binding sequence. A factor in adipocyte nuclear extracts that bound to this site in electrophoretic mobility shift assays was shown to be predominantly $\mathrm{C} / \mathrm{EBP} \alpha$ by supershifting with anti-C/ $\mathrm{EBP} \alpha$ antibodies. Furthermore, $\mathrm{C} / \mathrm{EBP} \alpha$ was found to transactivate $\mathrm{ob}$ promoter-reporter constructs in a manner that was abrogated by a mutation of this site. Thus, it appears that $\mathrm{C} / \mathrm{EBP} \alpha$ functions as a transcriptional activator of the obese gene during adipocyte differentiation.

Consistent with its role as a regulator of adipose homeostasis, leptin expression is regulated by adiposity. Under conditions of fasting, when adipose stores are being depleted, expression is strongly repressed. Conversely, when there is an energy surplus leptin expression is positively correlated with increased adipose mass, reaching very high levels in obese individuals. An increasing body of evidence indicates that expression of leptin is rigorously controlled by agents, e. g. hormones and cytokines, that respond to changes in energy status or stress. Effectors such as insulin and cAMP, known to modulate energy metabolism in response to changes in the global energy status, have 
been found to alter leptin expression by the adipocyte.

In culture, adipocyte cell lines, e.g. the 3T3-L1 and -F442A lines, express leptin mRNA at an exceedingly low level ( $<1 \%$ that in adipose tissue). A new approach for studying adipose development and regulation of adipose-specific genes in an in vivo context has recently been developed [6]. Green and Kehinde [7] showed that subcutaneous implantation of 3T3F442A cells into athymic (nude) mice gave rise to fat pads indistinguishable from endogenous white adipose tissue. Studies in which a $\beta$-galactosidase transgene was introduced prior to implantation have now demonstrated that the adipocytes in such fat pads are derived entirely from the implanted preadipocytes [6]. These adipocytes express levels of adiposespecific transcripts (including the leptin transcript) comparable to those by normal tissue adiocytes. Furthermore, the leptin gene in these implanted fat pads shows induction by glucocorticoid nearly identical to that observed in epididymal adipose tissue. Thus, it is possible that an additional factor (e.g. cytokine or extracellular matrix) or condition (e.g. neighbouring cell-cell contact) present in vivo, but lacking in cell culture medium, is necessary for adipose tissue-level expression of the obese gene. Since 3T3-F442A preadipocytes harbouring various types of transgenes can be used for implantation, this approach should be useful for studying the effects of transfected genes on differentiation "in vivo" and for the analysis of adipose-specific promoters that are not appropriately regulated in vitro.

\section{Michael Schwartz: Neuropeptide $Y$ and the control of body weight}

At the level of the central nervous system, the negative feedback control system implicates two hormones (Fig. 1), leptin and insulin, as humoral adiposity-related signals that provide afferent input to the central nervous system. The two signals arise from two separate anatomical sites in the periphery: leptin from adipose tissue and insulin from pancreatic islets. Within the brain, a hypothalamic pathway containing neuropeptide Y (NPY) is implicated as a potential mediator of afferent signals arising from peripheral tissue sites. However, it should be noted that the effective role of NPY seems to be in response to weight loss, as signalled by a fall in insulin and leptin levels. The far-reaching effects of NPY, considered an "anabolic hypothalamic effector", are seen following administration of NPY into the central nervous system. This results in a striking hyperphagic response, weight gain, increased fat stores and a reduction in sympathetic nervous system activity [8]. Under conditions of weight gain, however, where insulin and leptin signals are likely to be increased, it is likely

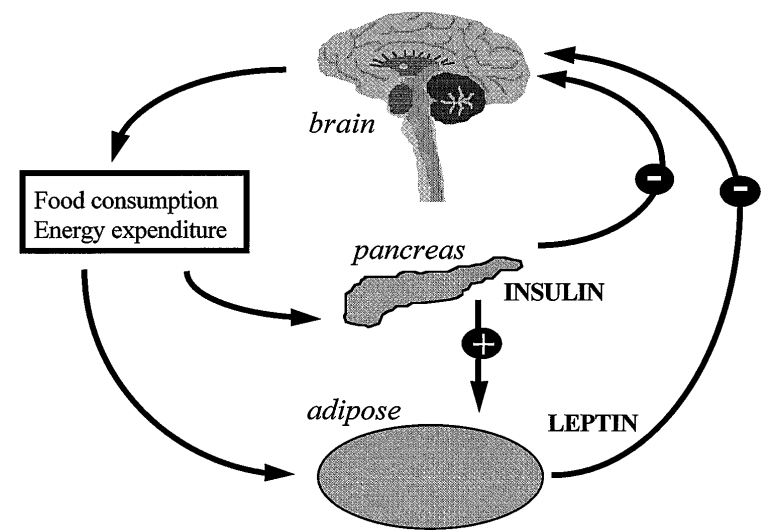

Fig. 1. Schematic representation of the energy balance homeostatic loop, demonstrating the brain (in the likely region of the arcuate nucleus of the hypothalamus) as a target organ for negative feedback afferent signals arising from pancreatic islets and adipose tissue

that NPY plays a less crucial role and there is evidence that alternate pathways (involving melanocyte stimulating hormone ( $\mathrm{MSH}$ ) and the agouti gene product) are involved. In other words, it should be made clear that NPY is not the sole regulatory pathway in the central nervous system through which peripheral signals act, even though overwhelming evidence suggests that the effects of NPY in the brain are consistent with a critical role in the response to weight loss. As a result, repeated NPY injection directly into the brain of rats can cause obesity in a matter of days. These central actions of NPY are believed to be mediated via the "Y ${ }_{5}$ " receptor that is concentrated in hypothalamic areas such as the paraventricular nuclei (PVN) [9]. For NPY to be implicated in energy homeostasis, however, it must be demonstrated that the relevant NPY system is activated when the animal enters a state of negative energy balance. The hypothalamic NPY pathway implicated in this response is comprised of neuronal cell bodies in the arcuate nucleus and their axonal projections to other hypothalamic areas such as the PVN [8]. As predicted, this hypothalamic pathway is activated by fasting, as measured by increased NPY mRNA levels in the arcuate nucleus and increased NPY release into the PVN. These observations focus attention on the question of which metabolic signals are important in triggering this activation of the NPY pathway.

Leptin is now implicated as an important controller of the hypothalamic NPY system. This conclusion is based on the observation that mice with both leptin deficiency (ob/ob) and leptin resistance, due to mutation of the leptin receptor $(\mathrm{db} / \mathrm{db})$, overexpress NPY in the hypothalamic arcuate nucleus. Moreover, leptin administration to ob/ob mice reduces expression of NPY mRNA in parallel with its effect in reducing food intake and body weight. In contrast, leptin affects neither food intake, body weight, nor NPY 
mRNA expression in the arcuate nucleus of $\mathrm{db} / \mathrm{db}$ mice. Moreover, the gene encoding the leptin receptor is expressed by arcuate nucleus neurons, suggesting that leptin may mediate inhibitory effects on NPY gene expression via a direct or indirect action on NPY neurons. Thus, it is not yet known whether the effect of leptin on the NPY system occurs directly on NPY neurons expressing leptin receptors or via an indirect route involving other neural circuits.

Recent studies with the NPY "knockout" mouse have added insight into the relationship between leptin and NPY. Surprisingly, these NPY-deficient mice maintain normal levels of body weight and exhibit normal patterns of food intake [10]. However, leptin-induced anorexia is heightened in mice with NPY deficiency. This very significant finding adds credence to the idea that NPY is not necessary for leptin to inhibit food intake. It is more likely that NPY signalling normally exerts effects that oppose those of leptin, and thus NPY removal potentiates leptin action within the brain. Conversely, NPY appears to be a major mediator of the response to leptin deficiency. This conclusion is based in part on studies of mice in which genetic deletion of NPY is introduced into the ob/ob background. In these animals, the obesity syndrome associated with leptin deficiency is substantially attenuated by a concomitant deficiency of NPY [11]. These observations suggest that in the absence of leptin, unrestrained activation of the NPY system in the hypothalamus leads to a variety of neuroendocrine and autonomic disturbances that play a major role in the resulting obesity syndrome. However, the persistence of some degree of obesity in the absence of NPY indicates that additional factors are involved in leptin's control of adiposity.

Insulin, secreted from pancreatic islet cells, is also implicated as an inhibitory regulator of the NPY system. Like leptin, insulin is secreted in proportion to adiposity, and it acts in the brain to suppress food intake and promote weight loss [12]. The importance of insulin's central signalling role in inhibiting the NPY system is supported by studies demonstrating that fasting-induced stimulation of NPY gene expression in the arcuate nucleus [12] and NPY release into the PVN [13] is inhibited by insulin administration. Moreover, insulin receptors are expressed in the brain and are concentrated in the arcuate nucleus [12]. These observations raise the possibility that insulin-deficient states such as fasting and uncontrolled diabetes stimulate food intake via a mechanism by which reduced insulin signalling in the brain activates the NPY system. This hypothesis is supported by studies in streptozotocin-induced diabetic rats. After cannulation of the third cerebral ventricle of these animals, it was shown that the ability of uncontrolled diabetes to increase food intake and hypothalamic NPY gene expression was attenuated by intracerebroventricular (ICV) insulin administration. This effect appeared to be specific for feeding behaviour, since the polydipsic response to diabetes was intact in ICV insulin-treated animals. Moreover, ICV insulin administration did not reduce the severe hyperglycaemia present in diabetic rats, demonstrating that insulin's effects were mediated directly in the brain and not via an improvement in the diabetic state. These studies suggest that insulin deficiency in the brain may lead to activation of the NPY system, which in turn may be an important mediator of diabetic hyperphagia. Since insulin-deficient diabetes also lowers leptin levels, it seems likely that combined insulin and leptin deficiency participate in this response.

\section{Conclusion}

In this rapidly evolving field, it is clear that leptin fits the criteria for a critical afferent signal arising from the adipose tissue fat mass and exerting its signal in the central nervous system in the hypothalamus. However, it is not clear whether this is the sole function of leptin; important action in other sites in the central nervous system or in peripheral tissue sites are also possible. There is evidence to suggest that insulin is also an important afferent signal acting on the same or similar regulatory pathways in the central nervous system. However, insulin's effects on the central nervous system, resulting in adaptive changes in eating behaviour and energy expenditure, are difficult to separate from its systemic effects on fuel metabolism. In the central nervous system, NPY appears to be an important mediator of the adaptive response to a state of negative energy balance. A reduction in negative feedback signalling provided by the hormones, insulin and leptin, is implicated in the activation of the NPY system. Conditions associated with defective leptin signalling induce pathological overexpression of NPY which appears to participate in the resulting obesity syndrome. Overactivity of the NPY system due to insulin and leptin deficiency may similarly contribute to the hyperphagic response to uncontrolled diabetes.

\section{Future directions and recommendations}

Further research is needed in several critical areas. It will be important to determine:

- How the central nervous system alters glucose metabolism in physiologic and pathologic states.

- The significance of centrally mediated regulation of energy homeostasis by insulin.

- The extent to which leptin acts in peripheral tissues.

- Which factors (e.g. insulin, etc.) are involved in the activation of leptin production and secretion by the adipocyte. 
- The levels, function and distribution of the different types of leptin receptors, particularly the long form, in peripheral tissues.

- The nature of the hypothalamic (and peripheral tissue) signalling system that exists downstream of leptin.

- The relative importance of primary and secondary leptin resistance in human forms of obesity.

\section{References}

1. Kennedy GC (1953) The role of depot fat in the hypothalamic control of food intake in the rat. Proc $\mathrm{R}$ Soc Lond (Biol) 140: 579-592

2. Zhang Y, Proenca R, Maffei M, Barone M, Leopold L, Friedman JM (1994) Positional cloning of the mouse obese gene and its human homologue. Nature 72: 425-432

3. Bray GA, York DA, Fisler JS (1989) Experimental obesity: a homeostatic failure due to defective nutrient stimulation of the sympathetic nervous system. Vit Horm 45: 1125

4. Coleman DL (1973) Effects of parabiosis of obese with diabetes and normal mice. Diabetologia 9: 294-298

5. MacDougald OA, Lane MD (1995) Transcriptional regulation of gene expression during adipocyte differentiation. Ann Rev Biochem 64: 345-373
6. Mandrup S, Loftus TM, MacDougald OA, Kuhajda F, Lane MD (1997) Leptin is expressed at in vivo levels by fat pads derived from subcutaneously implanted 3T3-F442A preadipocytes. Proc Natl Acad Sci USA 94: 4300-4305

7. Green H, Kehinde O (1979) Formation of normally differentiated subcutaneous fat pads by an established preadipose cell line. J Cell Phys 101: 169-172

8. Stanley BG (1993) Neuropeptide Y in multiple hypothalamic sites controls eating behavior, endocrine, and autonomic systems for energy balance. In: Colmers WF, Wahlestedt C (eds) The biology of neuropeptide $\mathrm{Y}$ and related peptides. Humana Press, Totowa, pp 457-509

9. Gerald C, Walker MW, Criscione L et al. (1996) A receptor subtype involved in neuropeptide-Y-induced food intake. Nature 382: 168-171

10. Erickson JC, Clegg KE, Palmiter RD (1996) Sensitivity to leptin and susceptibility to seizures of mice lacking neuropeptide Y. Nature 381: 415-418

11. Erickson JC, Hollopeter G, Palmiter RD (1996) Attenuation of the obesity syndrome of ob/ob mice by the loss of neuropeptide Y. Science 274: 1704-1707

12. Schwartz MW, Figlewicz DP, Baskin DG, Woods SC, Porte D Jr (1992) Insulin in the brain: a hormonal regulator of energy balance. Endocrinol Rev. 13: 387-414

13. Sahu A, Dube M, Phelps C, Sninsky C, Kalra P, Kalra S (1995) Insulin and insulin-like growth factor II suppress neuropeptide $\mathrm{Y}$ release from the nerve terminals in the paraventricular nucleus: a putative hypothalamic site for energy homeostasis. Endocrinology 136: 5718-5724 